\title{
ANÁLISE DE MERCADO DE RÓTULOS ALIMENTÍCIOS POR CONSUMIDORES DE GOIÂNIA
}

\author{
Market analysis of food labels by consumers of Goiânia
}

Análisis de mercado de rótulos alimenticios por consumidores de Goiânia

Fábio Santos da Silva*1, Thaís Cristina de Oliveira Pereira ${ }^{1}$, Yorrana Afonso Ramos do Carmo ${ }^{1}$, Adriana Régia Marques de Souza ${ }^{2}$

${ }^{1}$ Discente do Curso de Engenharia de Alimentos, Universidade Federal de Goiás, Goiânia, Brasil.

${ }^{2}$ Docente do Curso de Engenharia de Alimentos, Universidade Federal de Goiás, Goiânia, Brasil.

*Correspondência: Universidade Federal de Goiás, Avenida Esperança s/n - Campus Samambaia, Goiânia, Goiás, Brasil. CEP:74.690-900. E-mail fbsantos.silva@gmail.com

\section{RESUMO}

O rótulo tem por função orientar o consumidor sobre os constituintes dos alimentos. Tendo em vista as informações contidas nos rótulos, o objetivo desse trabalho foi realizar uma pesquisa sobre o hábito que as pessoas têm de ler rótulos, quais informações influenciam na compra e quais as maiores dificuldades encontradas pelos consumidores. Assim, realizou-se uma pesquisa de mercado em Goiânia (GO), com homens e mulheres de idades variadas, os quais responderam um questionário. Entre as pessoas entrevistadas, 39\% afirmaram ter o hábito de ler os rótulos. Mais da metade das pessoas que afirmaram ler rótulos, respondeu que as informações contidas são determinantes na hora de efetuar a compra, visto que $40 \%$ dos entrevistados levam em consideração a data de validade e $15 \%$ o teor de sódio. A maioria dos entrevistados declararam que as informações contidas nos rótulos não são claras e 38\% disseram ter dificuldades em ler letras pequenas. O estudo revelou que, apesar da maioria dos entrevistados afirmarem ler rótulos alimentícios, a data de validade e o teor de sódio são as informações mais relevantes.

Palavras-chave: Pesquisa, Embalagem, Cliente.

\section{ABSTRACT}

The purpose of the label is to guide the consumer about the constituents of food. Considering the information contained in the labels, the objective of this work was to conduct a survey about people's habit of reading labels, which information influences the purchase and which are the greatest difficulties encountered by consumers. Thus, a market research was conducted in Goiânia-GO, with men and women of different ages, who answered a questionnaire. Of the people interviewed, 39\% said they had a hab contained in them is decisive at the time of purchase, as $40 \%$ of respondents take into account the expiration date and $15 \%$ the sodium content. Most respondents stated that the information on the labels is unclear and 38\% said they had difficulty reading small print. The study found that although most respondents claimed to read food labels, the expiration date and sodium content are the most relevant information. it of reading the labels. More than half of those who claimed to read labels replied that the information.

Keywords: Research, Packing, Customer.

\section{RESUMEN}

La etiqueta tiene por función orientar al consumidor sobre los constituyentes de los alimentos. En cuanto a la información contenida en las etiquetas, el objetivo de este trabajo fue realizar una investigación sobre el hábito que las personas tienen que leer etiquetas, qué información influyen en la compra y cuáles son las mayores dificultades encontradas por los consumidores. Así, se realizó una investigación de mercado en Goiânia-GO, con hombres y mujeres de edades variadas, los cuales respondieron un cuestionario. Entre las personas entrevistadas, el 39\% 
afirmó tener el hábito de leer las etiquetas. Más de la mitad de las personas que afirman leer etiquetas, respondió que las informaciones contenidas son determinantes a la hora de efectuar la compra, ya que el $40 \%$ de los encuestados toma en consideración la fecha de caducidad y el 15\% el contenido de sodio. La mayoría de los interrogados declararon que la información contenida en las etiquetas no es clara y el 38\% dijo que tenían dificultades para leer letras pequeñas. El estudio reveló que, a pesar de que la mayoría de los encuestados afirman leer etiquetas alimentícias, la fecha de caducidad y el contenido de sodio son las informaciones más relevantes.

Descriptores: Búsqueda, Embalaje, Cliente.

\section{INTRODUÇÃO}

Pesquisa de mercado é a obtenção de informações junto ao consumidor, com a finalidade de orientar empresas a tomar decisões ou solucionar problemas. É a coleta de dados úteis para a atividade mercadológica e que podem resultar no conhecimento prévio das reações dos consumidores (Azevedo, 2015).

A legislação brasileira define rótulo como toda inscrição, legenda ou imagem, ou toda matéria descritiva ou gráfica, escrita, impressa, estampada, gravada, gravada em relevo ou litografada ou colada sobre a embalagem do alimento (Brasil, 2002). Essas informações permitem a identificação da origem do produto, suas características nutricionais, bem como o rastreamento do mesmo através do lote, sendo, portanto, uma questão de segurança da saúde do consumidor.

A rotulagem nutricional tem como objetivo principal de operar em benefício do consumidor, orientando o consumidor quanto à qualidade do produto. A Resolução ANVISA RDC 360/2003 Regulamento Técnico Sobre Rotulagem Nutricional de Alimentos Embalados determina a informação nutricional obrigatória de valor energético, carboidratos, proteínas, gorduras totais, gordura saturada, gordura trans, fibra alimentar e sódio, partindo de uma dieta de $2000 \mathrm{Kcal}$ ou $8400 \mathrm{KJ}$ diárias (Brasil, 2005). A rotulagem de alimentos deve, também, atender ao disposto no capítulo III do Decreto-Lei $n^{\circ} .986 / 69$, à Lei ${ }^{\circ} .10,674 / 2003$ relativa à informação prevista para o glúten.
Com o aumento de doenças relacionadas aos hábitos alimentares, verificou-se uma maior procura por uma boa alimentação. Diante disso, o rótulo se tornou um fator determinante na compra de alimentos, sendo que a rotulagem também é um modo de comparação entre dois produtos, propiciando ao consumidor a escolha de um alimento mais saudável (Enes e Slater, 2010).

As informações contidas nas embalagens dos alimentos são importantes para sua preservação e integridade, bem como para a segurança de consumidores que possuem algum tipo de restrição alimentar, ou mesmo para pessoas que seguem uma dieta. Considerando as diferentes necessidades de cada consumidor, é relevante a identificação das principais informações nos rótulos alimentares que os consumidores leem antes de comprarem o produto.

Este trabalho teve como objetivo realizar uma análise de mercado referente à leitura de rótulos alimentícios, destacando as maiores dificuldades para a sua compreensão e quais informações influenciam o consumidor antes de efetuar a compra.

\section{MATERIAIS E MÉTODOS}

Foi realizado um estudo de campo com levantamento de dados estatísticos a partir de um questionário semiestruturado (Figura 1) com dez perguntas fechadas e uma aberta, impresso e entregue aos entrevistados, obtendo 300 respostas. Os consumidores entrevistados nesta etapa foram selecionados por demonstrarem interesse no tema abordado e por apresentar disponibilidade de tempo. 
Foram selecionados dois lugares distintos na região de Goiânia: feira frutas e legumes na região do setor dos funcionários em Goiânia (GO) (120 entrevistados) e no restaurante universitário da Universidade Federal de Goiás (180 entrevistados).

Figura 1. Questionário aplicado na análise.

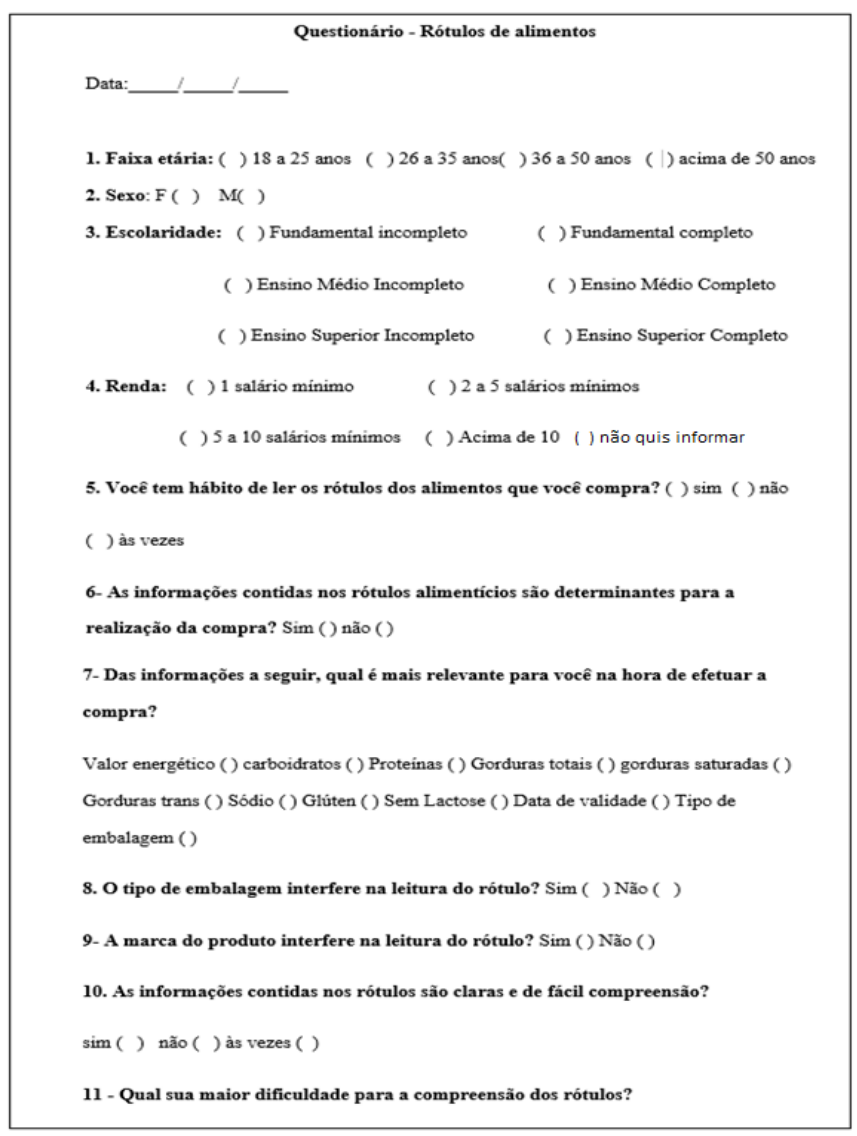

As respostas obtidas por meio do questionário foram tabuladas por meio da planilha eletrônica Microsoft Excel (2016) onde foi utilizada uma estatística descritiva, expondo os resultados mediante comparação gráfica do conjunto de dados.

\section{RESULTADOS E DISCUSSÃO}

O público entrevistado foi em maioria pessoas de 18 a 25 anos (Figura 2A), na sua maior parte do sexo feminino (Figura 2B), predominantemente de pessoas com escolaridade de ensino superior incompleto (Figura 2C). Para informação da renda, notou-se que a maioria optou por não informar sua renda (Figura 2D).
Figura 2. Perfil dos entrevistados sobre rótulos de alimentos, segundo faixa etária (A), sexo (B), escolaridade (C) e renda (D).

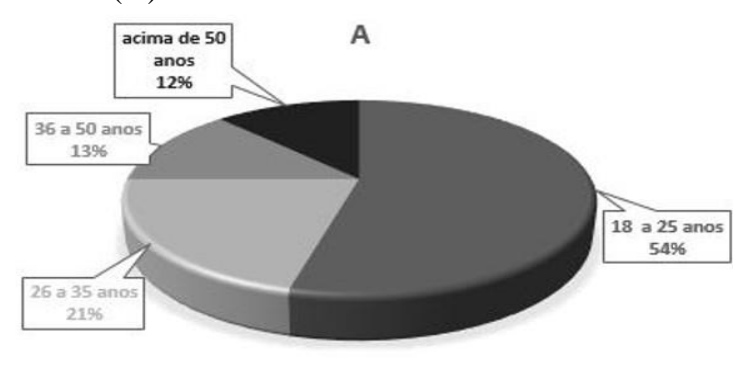

B
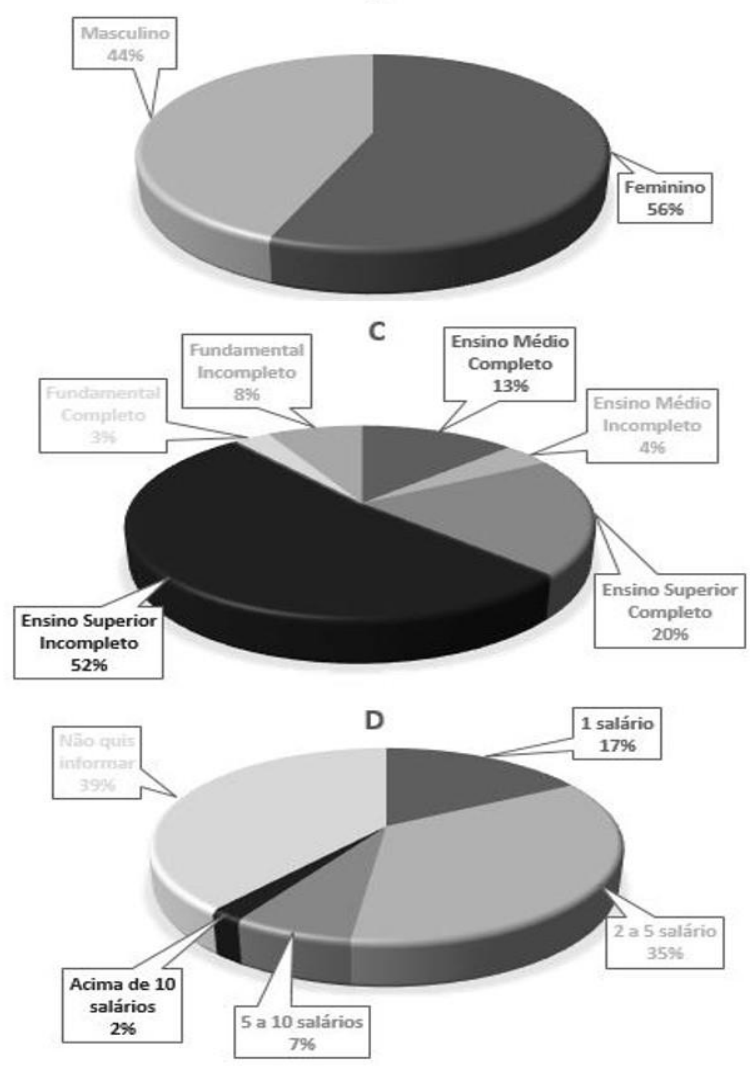

De acordo com a Figura 3, os entrevistados têm o hábito de ler os rótulos, contudo ainda corresponde menos da metade do total da amostra. Segundo Pesquisa do Datafolha (Cancian, 2016), encomendada pela Aliança de Controle do Tabagismo (ACT), organização que atua na área de promoção da saúde, $48 \%$ dos brasileiros não costumam ler as informações contidas nos rótulos, como ingredientes e tabela nutricional. A maioria dos entrevistados são pessoas entre 18 e 25 anos onde, segundo a pesquisa Global Nielsen (2016), Estilo de Vida das Gerações, $81 \%$ dos jovens estão dispostos a desembolsar mais para consumir alimentos mais saudáveis, acarretando assim, o interesse dos consumidores pelas informações 
contidas nos rótulos. Outro parâmetro de influência para a pergunta "Você tem o hábito de ler os rótulos dos alimentos que você compra?", foi a escolaridade (Figura 3B), visto que dos $39 \%$ entrevistados que afirmaram ter o costume de ler rótulos, $58 \%$ possuem ensino superior incompleto. De acordo com Cassemiro et al. (2008), pessoas com maior grau de instrução utilizam a informação nutricional. Monteiro et al. (2005) também encontraram uma relação direta e significativa entre hábito de leitura dos rótulos $\mathrm{e}$ escolaridade, corroborando com os resultados obtidos neste trabalho.

Figura 3. Leitura de rótulos alimentícios (A), segundo influência da escolaridade (B) e sexo (C) (D).

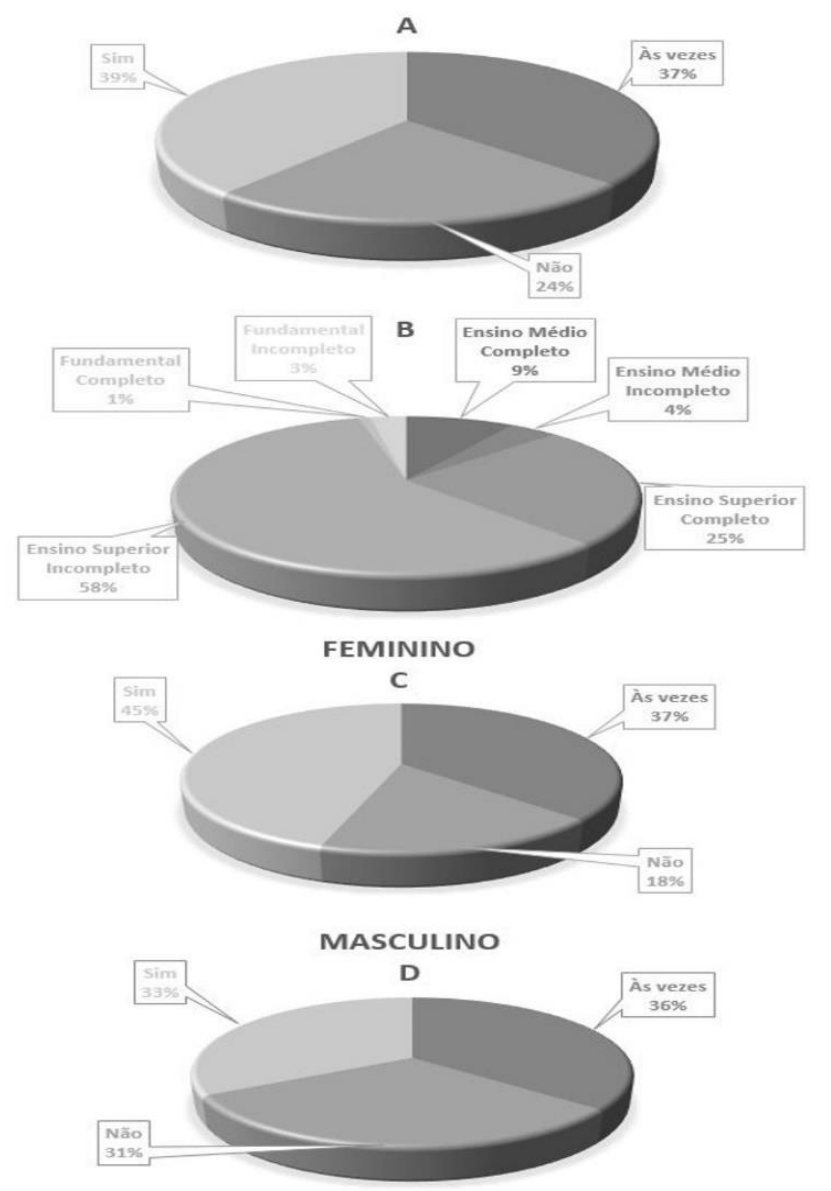

Em relação às pessoas que afirmaram ler os rótulos alimentícios, observou-se que as mulheres têm maior hábito de ler informações contidas nos rótulos (Figura 3C) em relação aos homens (Figura 3D). Segundo a pesquisa do Instituto Data Popular (Folha de São Paulo, 2013), 86\% dos maridos disseram que suas mulheres decidem as compras do supermercado.
De acordo com o portal do Instituto Brasileiro de Defesa do Consumidor (IDCE, 2017), a Anvisa estuda uma mudança nos rótulos dos alimentos com o objetivo de deixar as informações mais claras para o consumidor sobre ingredientes em excesso e efeitos que eles podem causar à saúde. As propostas iniciais que estão em discussão são baseadas em dois eixos principais: o uso do sistema de semáforo com cores, que sinalizam se algum ingrediente está em excesso, e o uso de símbolos com advertência sobre algum ingrediente em excesso que pode fazer mal a saúde.

Em relação à questão que avaliou se as informações contidas nos rótulos alimentícios são determinantes para a realização da compra, a maior parte dos entrevistados afirmou ser influenciada por essas informações no âmbito da compra (Figura 4A), das quais levam em consideração algumas informações importantes: valor energético, carboidratos, proteínas, gorduras totais, gorduras saturadas, gorduras trans, sódio, glúten, sem lactose, data de validade e o tipo de embalagem (Figura 4B).

Dentre as opções, a data de validade foi o fator mais observado durante a leitura do rótulo, seguido da porcentagem de sódio e, em terceiro lugar, levam em consideração a quantidade de carboidrato do produto na hora de efetivar a compra (Figura 4B).

Segundo a Anvisa (2005), quando o prazo de validade for menor do que três meses, o fabricante é obrigado a colocar no rótulo apenas o mês e o dia em que o produto foi fabricado. Por sua vez, o sódio é utilizado na indústria com função de conservação de alimentos, assim, alimentos como embutidos, enlatados, queijos e salgadinhos trazem em sua composição grandes quantidades de sal (Costa e Machado, 2010). Segundo o Portal Brasil (2015), o excesso de sódio, está associado ao desenvolvimento da hipertensão arterial, de doenças cardiovasculares, renais e outras, que estão entre as primeiras causas de internações e óbitos no Brasil e no mundo. O sal é um 
ingrediente presente em quase todos os alimentos, principalmente aqueles industrializados, e desempenha importante papel em termos de propriedades funcionais e sensoriais: confere textura; em produtos doces, sua presença em pequenas quantidades realça o sabor doce; ajuda na estabilização da cor, além de proteger do crescimento microbiano (sais de cura); alguns produtos são produzidos em salmoura, e adição de sal em enlatados aumenta a preservação e confere sabor; em aperitivos (snacks), a aplicação de cristais de cloreto de sódio na superfície do produto proporciona atrativo adicional (Aditivos e Ingredientes, 2011).

Figura 4. Relevância das informações contidas nos rótulos no âmbito da compra (A) e informações mais importantes (B).

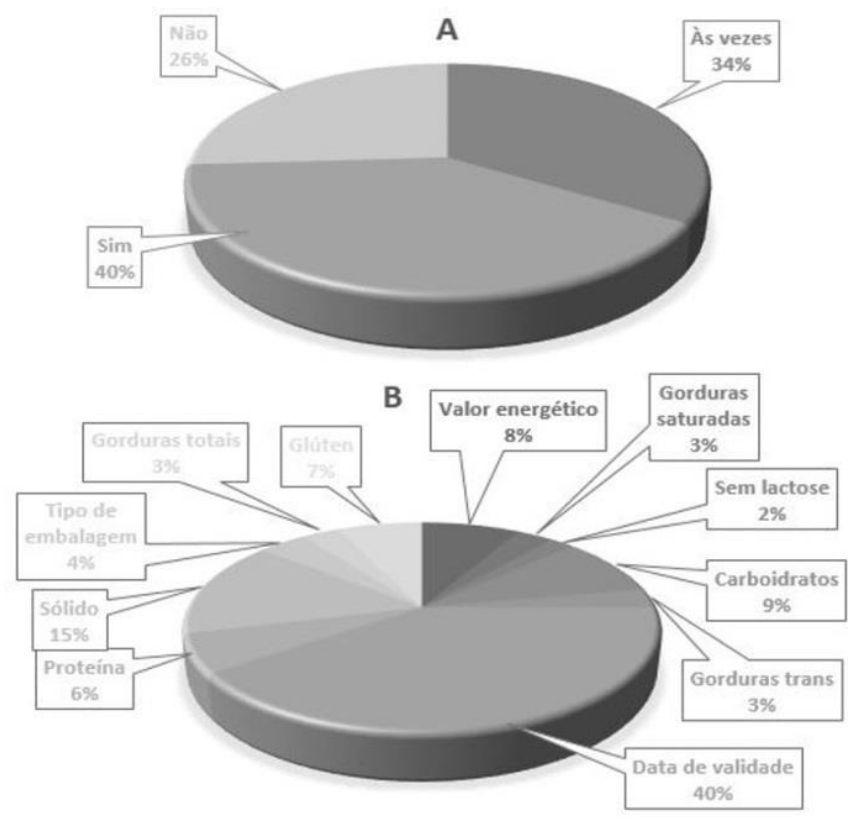

Para a pergunta "O tipo de embalagem interfere na leitura do rótulo?", os entrevistados em sua preponderância afirmaram que a embalagem interfere na leitura do rótulo (Figura 5).
Figura 5. Interferência da embalagem na leitura do rótulo.

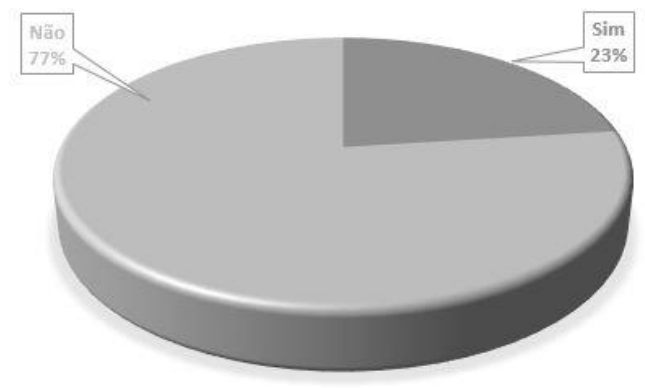

Comentários durante a pesquisa justificam a interferência da embalagem no momento da leitura, principalmente quando ela é muito chamativa, transparente, pequena ou quando seu rótulo prioriza sua logomarca.

Em relação à pergunta "A marca do produto interfere na leitura do rótulo?", mais da metade dos entrevistados admitiram que a marca influencia no momento da compra (Figura 6A). A prevalência dos homens que responderam sim a essa pergunta possui renda entre 2 a 5 salários mínimo (6B). Já as mulheres, em sua maioria, optaram por não informar sua renda (6C). Em 1940, os fabricantes perceberam que os consumidores se relacionavam com a marca além do próprio produto. Então, começaram a associar seus produtos a outros tipos de valores, como por exemplo, status e poder aquisitivo. Isso deu início à prática conhecida atualmente como branding, na qual percebeu-se que os consumidores compram mais que produtos, compram marcas (Francischelli, 2009).

Comentários observados durante a pesquisa foram que alguns consumidores já estavam acostumados a comprar certas marcas, por consequência não realizavam a leitura dos seus rótulos. 
Figura 6. Influência da marca no poder de compra (A), segundo a renda de homens (B) e mulheres (C).

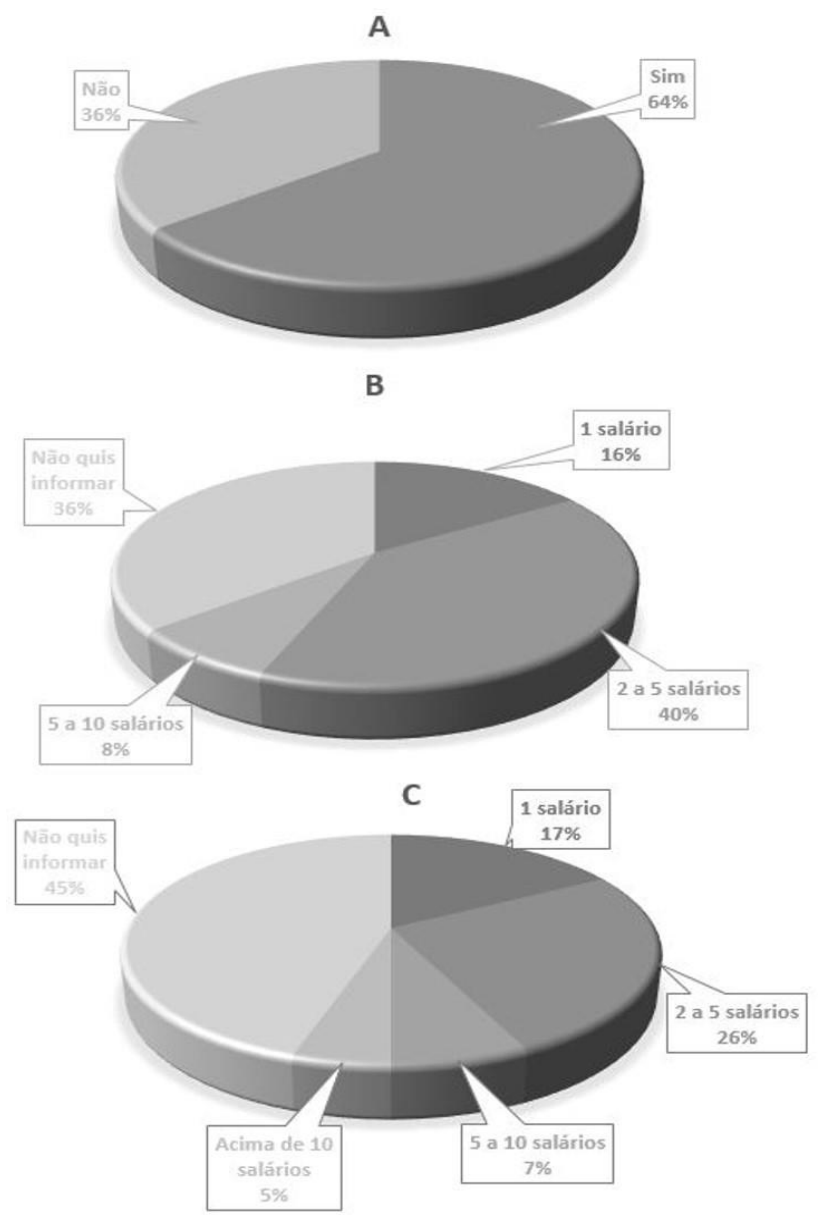

Para a questão "As informações contidas nos rótulos são claras e de fácil compreensão", a maioria dos entrevistados declarou que as informações nem sempre são claras e de fácil compreensão (Figura 7A), ou seja, a maioria possui alguma dificuldade na interpretação dos mesmos. Segundo o Instituto Brasileiro de Defesa do Consumidor (Brasil, 2017), a Anvisa almeja que os rótulos de alimentos tenham dados mais claros. Essa medida terá um impacto sobre todos os alimentos industrializados que não devem ser consumidos em excesso, conscientizando o consumidor.

Notou-se que a maioria dos entrevistados possui complicações em compreender termos técnicos, seguido daqueles que têm dificuldades em ler letras pequenas (Figura 7B). De acordo com a Empresa Brasil de Comunicação (2015), 6,2\% da população brasileira tem algum tipo de deficiência, dentre elas a visual é a mais representativa e atinge 3,6\% dos brasileiros. O grau intenso da limitação impossibilita $16 \%$ dos deficientes visuais de realizar atividades habituais.

Figura 7. Facilidade de compreensão de rótulos (A) e maiores dificuldades na leitura (B).

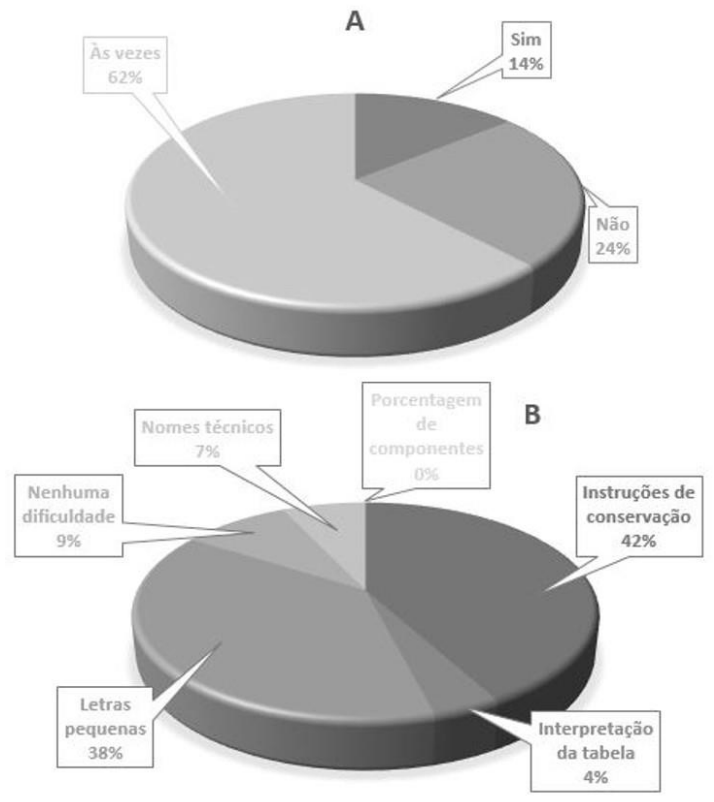

\section{CONCLUSÃO}

A leitura dos rótulos ainda é algo primário e insuficiente no âmbito da compra, onde as informações relacionadas à data de validade e teor de sódio são mais importantes para o consumidor. Os rótulos, na sua maioria, não possuem informações claras e de fácil compreensão, sendo que os termos técnicos e letras pequenas são as maiores dificuldades encontradas pelos entrevistados. Logo, é pertinente a proposta da Anvisa em deixar os rótulos mais claros para os consumidores, visto que há uma grande dificuldade de interpretação das informações por parte dos entrevistados de Goiânia (GO).

Todos os autores declararam não haver qualquer potencial conflito de interesses referente a este artigo.

\section{REFERÊNCIAS}

ADITIVOS E INGREDIENTES. 2011. O Sal e seus Substitutos. Disponível em: 
http://www.insumos.com.br/aditivos_e_ingredientes/ materias/246.pdf. Acesso em: 6/10/2017.

AZEVEDO, G C. I. 2015. Pesquisa de Mercado: entenda o que é uma pesquisa de mercado. Disponível

em: http://www.bibliotecas.sebrae.com.br/chronus/ARQU IVOS_CHRONUS/bds/bds.nsf/24131C962E2F9B6C 0325714700683043/\$File/NT00031FF6.pdf. Acesso em: 18/04/2017.

BRASIL, Anvisa - Agência Nacional de Vigilância Sanitária. 2002. Resolução RDC $\mathbf{n}^{\mathbf{0}} \mathbf{2 5 9}$, de 20 de setembro de 2002 sobre Regulamento Técnico sobre Rotulagem de Alimentos Embalados. Disponível em: http://www.ibravin.org.br/admin/arquivos/informes/1 455824267-1ed.pdf. Acesso em: 15/04/2017.

BRASIL, Anvisa - Agência Nacional de Vigilância Sanitária. 2005. Resolução RDC $\mathbf{n}^{\mathbf{0}} \mathbf{3 0 2}$, de 13 de outubro de 2005. Disponível em: http://portal.anvisa.gov.br/documents/10181/2718376 /RDC_302_2005_COMP.pdf/7038e853-afae-4729948b-ef6eb3931b19. Acesso em: 17/05/2017.

BRASIL, Anvisa - Agência Nacional de Vigilância Sanitária. 2017. Queremos rótulos que ajudem o consumidor a comer bem. Disponível em: http://portal.anvisa.gov.br/noticias/-

/asset_publisher/FXrpx9qY7FbU/content/queremosrotulos-que-ajudem-o-consumidor-a-comer-

bem/219201?inheritRedirect $=$ false $\&$ redirect $=\mathrm{http} \% 3$ A\%2F\%2Fportal.anvisa.gov.br\%2Fnoticias\%3Fp_p_i d\%3D101_INSTANCE_FXrpx9qY7FbU\%26p_p_lif ecycle\%3D0\%26p_p_state\%3Dnormal\%26p_p_mod e\%3Dview\%26p_p_col_id\%3Dcolumn-

2\%26p_p_col_count\%3D1\%26_101_INSTANCE_F Xrpx9qY7FbU_advancedSearch\%3Dfalse\%26_101_ INSTANCE_FXrpx9qY7FbU_keywords\%3D\%26_1 01_INSTANCE_FXrpx9qY7FbU_delta\%3D20\%26p _r_p_564233524_resetCur\%3Dfalse\%26_101_INST ANCE_FXrpx9qY7FbU_cur\%3D5\%26_101_INSTA NCE_FXrpx9qY7FbU_andOperator\%3Dtrue. Acesso em: 06/10/2017.

BRASIL, Anvisa - Agência Nacional de Vigilância Sanitária. 2005. Manual Nutricional Obrigatória: Manual de Orientação às Indústrias de Alimentos. Disponível em: http://portal.anvisa.gov.br/documents/33916/389979/ Rotulagem+Nutricional+Obrigat\%C3\%B3ria+Manua $1+$ de+Orienta\% C3\%A7\%C3\%A3o+\%C3\%A0s+Ind $\%$ C3\%BAstrias+de+Alimentos/ae72b30a-07af-42e28b76-10ff96b64ca4. Acessado em: 15/04/2017.

CANCIAN, N. 2016. Quase metade dos brasileiros não lê rótulos de alimentos. Disponível em: http://www1.folha.uol.com.br/equilibrioesaude/2016/ 11/1828576-quase-metade-dos-brasileiros-nao-lerotulos-de-alimentos.shtml. Acesso em: 6/10/2017.

CASSEMIRO, I. A.; COLAUTO, N. B.; LINDE, G. A. Rotulagem nutricional: quem lê e por quê?. Arquivo Ciência Saúde UNIPAR, Umuarama, v. 10, n. 1, p. 9-16, 2008.

COSTA, F. P.; MACHADO, S. H. O consumo de sal e alimentos ricos em sódio pode influenciar na pressão arterial das crianças?. Ciência \& Saúde Coletiva, v. 15, p. 1383-1389, 2010.

EBC, Empresa Brasil de Comunicação. 2015. IBGE: 6,2\% da população possui algum tipo de deficiência. Disponível em: http://agenciabrasil.ebc.com.br/geral/noticia/201508/ibge-62-da-populacao-tem-algum-tipo-dedeficiencia. Acesso em: 16/10/2017.

ENES, C. C.; SLATER, B. Obesidade na adolescência e seus principais fatores determinantes. Revista Brasileira de epidemiologia, v. 13, p. 163-171, 2010.

FRANCISCHELLI, P. A importância da marca no processo de decisão de compra de calçados esportivos para a população de baixa renda. Tese de Doutorado. Fundação Getúlio Vargas - FGV; 2009

IDCE, Instituto Brasileiro de Defesa do Consumidor. 2017. Mudança em rótulos de comida abre 'guerra'. Disponível em: https://idec.org.br/idec-naimprensa/mudanca-em-rotulos-de-comida-abreguerra. Acesso em: 14/12/2017.

MICROSOFT. 2016. Microsoft Excel. Disponível em: https://products.office.com/pt-br/excel. Acesso em: 06/02/2017.

MONTEIRO, R. A.; COUTINHO, J. G.; RECINE, E. Consulta aos rótulos de alimentos e bebidas por frequientadores de supermercados em Brasília, Brasil. Revista Panamericana de Salud Pública, v. 18, p. 172-177, 2005.

NIELSEN. 2016. Estilo de Vida das Gerações: quanto a idade influencia nosso comportamento?. Disponível em: http://www.nielsen.com/br/pt/insights/news/2016/Esti los-de-vida-das-geracoes-globais-quanto-a-idadeinfluencia-nosso-comportamento.html. Acesso em: 16/10/2017.

PORTAL BRASIL. 2015. Hipertensão atinge mais de 30 milhões de pessoas no país. Disponível em: http://www.brasil.gov.br/saude/2015/04/hipertensaoatinge-mais-de-30-milhoes-de-pessoas-no-pais.

Acesso em: 08/10/2017. 
SÃO PAULO, FOLHA DE. 2013. Mulheres decidem compras da família. Disponível em: http://www1.folha.uol.com.br/mercado/2013/03/1241 880-mulheres-decidem-compras-da-familia-dizpesquisa.shtml. Acesso em: 16/10/2017. 